\title{
Enhanced endosomal escape by photothermal activation for improved small interfering RNA delivery and antitumor effect
}

This article was published in the following Dove Press journal:

International Journal of Nanomedicine

Xi Yang, ${ }^{1,2, *}$ Bo Fan, ${ }^{3, *}$ Wei

Gao, ${ }^{4,5, *}$ Liping Li, ${ }^{2}$ Tingting

$\mathrm{Li}^{3}{ }^{3}$ Jinghua Sun, ${ }^{2}$ Xiaoyang

Peng, ${ }^{2}$ Xiaoyan $\mathrm{Li}^{2}{ }^{2}$ Zhenjun

Wang, ${ }^{2}$ Binquan Wang, ${ }^{4,5}$

Ruiping Zhang, ${ }^{2}$ Jun $\mathrm{Xie}^{1}$

'Department of Biochemistry and Molecular Biology, Shanxi Medical

University, Taiyuan, Shanxi, People's

Republic of China; ${ }^{2}$ Imaging Department

of Shanxi Provincial Cancer Hospital,

Shanxi Medical University, Imaging

Department of Shanxi Medical

University, Taiyuan, Shanxi, People's

Republic of China; ${ }^{3}$ Department of

Pharmacy, School of Pharmacy, Shanxi

Medical University, Taiyuan, Shanxi,

People's Republic of China; ${ }^{4}$ Department of Otolaryngology, Head and Neck

Surgery, The First Hospital, Shanxi

Medical University, Taiyuan, Shanxi,

People's Republic of China; ${ }^{5}$ Shanxi Key

Laboratory of Otorhinolaryngology

Head and Neck Cancer, Taiyuan, Shanxi,

People's Republic of China

*These authors contributed equally to this work
Correspondence: Ruiping Zhang Imaging Department of Shanxi Provincial Cancer Hospital, Shanxi Medical University, Imaging Department of Shanxi Medical University, No. 3 ZhiGongXinCun, Taiyuan 03000I, Shanxi, People's Republic of China Email zrp_7I42@I63.com

Jun Xie

Department of Biochemistry and Molecular Biology, Shanxi Medical University, No. 3

ZhiGongXinCun, Taiyuan 03000I, Shanxi,

People's Republic of China

Email xiejun1968@I26.com
Background: Effective endosomal escape is still a critical bottleneck for intracellular delivery of small interfering RNAs (siRNAs) to maximize their therapeutic efficacy. To overcome this obstacle, we have developed a photothermally triggered system by using the near-infrared (NIR) irradiation to achieve "on-demand" endosomal escape and subsequent siRNA release into cytoplasm.

Materials and methods: Herein, the poly-L-lysine (PLL) was successfully conjugated with melanin to obtain melanin-poly-L-lysine (M-PLL) polymer as a siRNA vehicle. The melanin was an efficient photothermal sensitizer, and the positive pendant amino groups of PLL could condense siRNAs to form stable complexes by electrostatic interactions.

Results and discussion: Inspired by its excellent photothermal conversion efficiency, the melanin was first involved in the siRNA delivery system. Confocal laser scanning microscopic observation revealed that after cellular uptake the photothermally induced endosomal escape could facilitate siRNAs to overcome endosomal barrier and be delivered into cytoplasm, which resulted in significant silence in the luciferase expression over the NIR- and melanin-free controls. Moreover, the anti-survivin siRNA-loaded M-PLL nanoparticles displayed great inhibitory effect on 4T1 tumor growth in vitro and in vivo.

Conclusion: These findings suggest that the M-PLL-mediated siRNA delivery is a promising candidate for therapeutic siRNA delivery and shows improved effect for cancer therapy via enhanced endosomal escape.

Keywords: melanin, poly-L-lysine, photothermal effect, endosomal escape, siRNA delivery

\section{Introduction}

Small interfering RNA (siRNA) has provided a potent strategy for cancer treatment due to its high specificity to silence target genes. Recent progresses in non-viral vectors based on various cationic materials make it possible to overcome the poor stability and inefficient cellular uptake of siRNAs. ${ }^{1,2}$ But effective endosomal escape has so far remained challenging to release siRNAs into the cytoplasm where they achieve therapeutic effects. ${ }^{3,4}$ To overcome this obstacle, some strategies have been developed to disrupt the endosomal membrane, such as the application of endosomolytic reagents (chloroquine), cationic polymers, and photochemistry-internalization (PCI) technique. ${ }^{5-7}$ Among these strategies, the exploitation of external stimuli to trigger "on-demand" drug release has received considerable attentions, ${ }^{8,9}$ especially nearinfrared (NIR) light, which exhibits attractive spatially and temporally controlled cargo release but exhibits less damage and deeper tissue penetration. ${ }^{10-12}$ 
In this study, we developed a photothermally triggered system by using the NIR irradiation to achieve "on-demand" endosomal escape and subsequent siRNA release into cytoplasm. ${ }^{9,13-17}$ Herein, melanin, a naturally distributed and biocompatible pigment, was used as an efficient photothermal sensitizer. ${ }^{18-20}$ Melanin could generate heat under NIR light due to its strong absorption in both far-infrared and NIR band. ${ }^{21,22}$ Inspired by its excellent photothermal conversion efficiency, we first attempt to involve melanin in the siRNA delivery system. We successfully conjugated melanin with poly-L-lysine (PLL) to obtain melanin-poly-L-lysine (M-PLL) copolymer, and the positive pendant amino groups of PLL were utilized to condense siRNAs to form stable complexes by electrostatic interactions. The physicochemical feature and cytotoxicity of both M-PLL and complexed nanoparticles were well characterized. Then, after the entry of nanoparticles into the tumor cells by endocytosis, NIR light would initiate heat generation to induce the rupture of endosomal membranes and subsequently facilitate siRNA delivery into cytoplasm. As a result, enhanced gene silencing efficiency was obtained by effective endosomal escape. Furthermore, survivin-targeted siRNA was loaded with M-PLL to prepare complexes for cancer treatment. By exploring the photothermal effect for enhanced gene delivery and improved silencing efficacy, this study investigated the antitumor therapy of M-PLL/siRNA in vitro and in vivo.

\section{Materials and methods Materials}

Melanin was obtained from Sigma-Aldrich (St Louis, MO, USA). PLL and bicinchoninic acid assay (BCA) kits were bought from Solarbio (Beijing, People's Republic of China). Enzyme-linked immunosorbent assay (ELISA) kit was purchased from Cloud-Clone Corporation (Wuhan, People's Republic of China). Calcein acetoxymethyl ester and propidium iodide (calcein-AM/PI) Double Stain Kit was purchased from YEASEN (Shanghai Yeasen Biotechnology Co. Ltd., Shanghai, People's Republic of China). The Cell Counting Kit-8 (CCK8) was supplied by DOJINDO Molecular Technologies, Inc. (Shanghai, People's Republic of China), and Luciferase assay kit was from Promega Corporation (Fitchburg, WI, USA). All products of siRNA including FAM-siRNA and siRNA

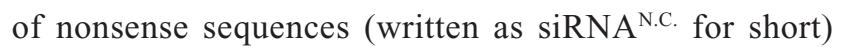
were provided by GenePharma Company (Shanghai, People's Republic of China). The surviving-targeted siRNA (siRNA ${ }^{\text {Sur: }}$ 5'-GCAUUCGUCCGGUUGCGCUTT-3') and luciferase-targeted siRNA (siRNA ${ }^{\text {Luc }}$ : 5'-CUUACGCUG AGUACUUCGATT-3') were also synthesized by GenePharma Company (Shanghai, People's Republic of China).

\section{Cell culture}

4T1 cells were obtained from the Department of Pathology, Institute of Medicinal Biotechnology, Peking Union Medical College. The cells were incubated in RPMI 1640 medium supplemented with $10 \%$ fetal bovine serum (FBS) at $37^{\circ} \mathrm{C}$ in humidified air containing $5 \% \mathrm{CO}_{2}$. Cells used in all experiments were in the logarithmic phase of growth.

\section{Animals}

Female Balb/c mice were 6-8 weeks old and obtained from Animal Center of Shanxi Medical University (Taiyuan, People's Republic of China). All animal experiments were performed in conformity to the institutional guidelines and ethically approved by the Institutional Animal Use and Care Committee of Shanxi Medical University (approval no 2016LL141) and complied with the US Guide for the Care and Use of Laboratory Animals 8th Edition 2011. ${ }^{31}$

\section{Synthesis and characterization of M-PLL}

The ultrasmall melanin nanoparticle (MNP) was first synthesized according to a previous method. ${ }^{23}$ In short, $20 \mathrm{mg}$ primitive melanin granule was dissolved in $4 \mathrm{~mL} 0.1 \mathrm{M}$ $\mathrm{NaOH}$ solution under vigorous stirring. Then, $0.1 \mathrm{M}$ hydrochloric acid solution was dropped into the alkaline melanin solution under sonication to adjust the $\mathrm{pH}$ value to 7.0. The solution was further centrifuged in a centrifugal-filter (MWCO $30 \mathrm{kDa}$ ) and washed with deionized water for five circles to remove the produced $\mathrm{NaCl}$.

To synthesize the polymer of M-PLL, the obtained MNP solution was adjusted to $\mathrm{pH} 9.0$ by adding $\mathrm{NaOH}$ solution (0.1 M). ${ }^{23}$ Next, $2 \mathrm{mg} \mathrm{mL}{ }^{-1}$ PLL aqueous solution was added dropwise into $1 \mathrm{mg} \mathrm{mL}^{-1} \mathrm{MNP}$ solution and followed by continuously and vigorously stirring for $24 \mathrm{~h}$ at room temperature. Finally, the obtained solution was transferred into a centrifugal filter unit (MWCO 30,000; EMD Millipore, Billerica, MA, USA), centrifuged at 4,000 rpm for $30 \mathrm{~min}$ and purified by deionized water. The product was further freeze-dried and characterized by Fourier transform-infrared (FT-IR) spectrometer and ${ }^{1} \mathrm{H}-\mathrm{NMR}$ to confirm the successful formation of M-PLL. To investigate the photothermal property of M-PLL polymer, the M-PLL was solved in phosphate-buffered solution (PBS) with a concentration of $0.75 \mathrm{mg} \mathrm{mL}^{-1}$ for UV detection and irradiated by NIR laser $\left(\lambda=808 \mathrm{~nm}\right.$ and the output power of $\left.1.5 \mathrm{~W} \mathrm{~cm}^{-2}\right)$.

\section{Preparation and characterization of M-PLL/siRNA nanoparticles (M-PLL-NPs)}

The solutions of M-PLL and siRNAs were complexed in equal volume, followed by vortexed for $30 \mathrm{~s}$ and standing 
for $20 \mathrm{~min}$ at room temperature. The particle size and zeta potential of M-PLL-NPs were measured by dynamic light scattering (DLS) method using Malvern Zetasizer Nano ZS90 (Malvern Instruments Ltd., Worcestershire, UK). The morphology image was acquired by transmission electron microscope (TEM) (JEM-2100F).

\section{Gel retardation assay}

The M-PLL solution of different concentrations complexed with $1 \mu \mathrm{g}$ siRNA at a weight ratio varying from 0 to 10 . Followed by blending with loading buffer, the M-PLL/ siRNA complexes were loaded onto $4 \%$ agarose gel and electrophoresed at $120 \mathrm{~V}$ for $20 \mathrm{~min}$. Then, the gel was stained with ethidium bromide solution for $30 \mathrm{~min}$. The UV imaging system was performed to investigate the loading capacity of M-PLL polymer.

\section{In vitro cytotoxicity assay}

The CCK8 assay was carried out to estimate the biosafety of melanin and M-PLL polymer and investigate antiproliferation efficacy of M-PLL-NPs on 4T1 cells. First, $100 \mu \mathrm{L}$ complete medium containing $5 \times 10^{3}$ cells was added into each well of 96-well plates. After adherence, cells were incubated with M-PLL polymer $\left(0-200 \mu \mathrm{g} \mathrm{mL}^{-1}\right)$ for $24 \mathrm{~h}$. Then, the old medium was discarded, fresh culture medium containing $10 \mu \mathrm{L}$ of CCK 8 reactant was put into each well and incubated at $37^{\circ} \mathrm{C}$ for $2 \mathrm{~h}$. Finally, the procedure was carried out according to the instruction of CCK8 assay. The absorbance per well was read at $450 \mathrm{~nm}$ to calculate the cell viability. The viability of cells not treated was deemed as $100 \%$. In addition, the cytotoxicity or anti-proliferation efficacy of M-PLL-NPs containing $100 \mathrm{nM}$ siRNA ${ }^{\text {N.C. }}$ or siRNA ${ }^{\text {Sur }}$ at different conditions (with or without NIR irradiation at $808 \mathrm{~nm}$ and $1.5 \mathrm{~W} \mathrm{~cm}^{-2}$ for various durations) was also studied as described previously.

\section{Survivin protein assay}

$4 \mathrm{~T} 1$ cells were seeded into 12 -well plates. After treated with M-PLL-NPs containing $100 \mathrm{nM}$ siRNA ${ }^{\text {Sur }}$ at different conditions (with or without NIR irradiation at $808 \mathrm{~nm}$ and $1.5 \mathrm{~W} \mathrm{~cm}^{-2}$ for seconds), cells were washed with PBS and maintained in fresh medium containing 10\% FBS for another $44 \mathrm{~h}$. Then, cells were collected, and survivin protein expression was quantitatively determined in cell lysates with a commercial survivin ELISA kit. Furthermore, the survivin protein levels were normalized by determining the protein concentration with BCA.

\section{Cell apoptosis assay}

4T1 cells were seeded into the 12-well plates and treated with naked siRNA, M-PLL/siRNA ${ }^{\text {N.C. }}$, or M-PLL/siRNA ${ }^{\text {Sur }}$ for $4 \mathrm{~h}$. The M-PLL/siRNA ${ }^{\text {N.C. }}$ and M-PLL/siRNA ${ }^{\text {Sur }}$ groups were then irradiated with laser at $808 \mathrm{~nm}$ and $1.5 \mathrm{~W} \mathrm{~cm}^{-2}$ for $180 \mathrm{~s}$. After replaced with fresh medium with $10 \% \mathrm{FBS}$ and cultured for another $20 \mathrm{~h}$, cells were harvested, washed twice with PBS, stained with Annexin V-FITC/PI according to the protocol, and finally analyzed by FACS Calibur flow cytometry for $30 \mathrm{~min}$.

\section{Confocal laser scanning microscopy (CLSM) analysis}

The cells were planted into the small glass dish with a density of $2 \times 10^{5}$ and cultured for $24 \mathrm{~h}$. After treated with naked FAMsiRNA or M-PLL/FAM-siRNA in a FBS-free medium for $4 \mathrm{~h}$, cells were (or not) irradiated by NIR light at $808 \mathrm{~nm}$ and $1.5 \mathrm{~W} \mathrm{~cm}^{-2}$ for $180 \mathrm{~s}$. Afterward, cells were incubated with LysoTracker Red DND-99 at $37^{\circ} \mathrm{C}$ for $1 \mathrm{~h}$ and fixed with $4 \%$ paraformaldehyde for $10 \mathrm{~min}$. After staining the nuclei with DAPI, samples were observed by a confocal laser scanning microscope (FV1000; Olympus Corporation, Tokyo, Japan).

\section{Calcein-AM/PI assay}

Cell viability of photothermal cytotoxicity was also estimated by using calcein-AM/PI staining method. ${ }^{22,24} 4 \mathrm{~T} 1$ cells were incubated in 96-well plates at a density of $5 \times 10^{3}$ cells/well and allowed for adherence. The cells were dealt with naked siRNA or M-PLL/siRNA ${ }^{\text {Sur }}$ for $4 \mathrm{~h}$ and then irradiated with a laser at $808 \mathrm{~nm}\left(1.5 \mathrm{~W} \mathrm{~cm}^{-2}\right)$ for $180 \mathrm{~s}$. After incubation for another $20 \mathrm{~h}$, cells were stained to differentiate live or dead cells by using calcein-AM and PI doubled stain kit and then imaged with a confocal laser scanning microscope.

\section{Luciferase gene expression assay}

$4 \mathrm{~T} 1^{\mathrm{Luc}}$ cells were seeded into the 24 -well plate and incubated for $24 \mathrm{~h}$ later. Next, cells were transfected with naked siRNA ${ }^{\text {Luc }}$ or M-PLL/siRNA ${ }^{\text {Luc }}$ for $4 \mathrm{~h}$, followed by NIR irradiation at $808 \mathrm{~nm}\left(1.5 \mathrm{~W} \mathrm{~cm}^{-2}\right)$ for $180 \mathrm{~s}$. PBS, lipofectamine

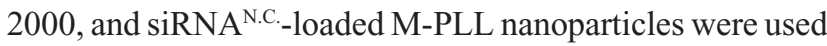
as controls. After $4 \mathrm{~h}$ coculture, the transfection medium was substituted by fresh medium. Another $44 \mathrm{~h}$ culture later, cells were treated according to the luciferase assay protocol. The luciferase activity in each well was determined on a SpectraMax i3x microplate reader (Molecular Devices LLC, Sunnyvale, CA, USA), and the protein concentration of cell extracts was detected by using commercial BCA protein kit at the same time. Then, the relative luciferase expression was calculated by comparing the relative light units (RLU) of the administration groups to the untreated control group.

\section{In vivo antitumor effect experiments}

To test the anticancer effect in vivo, $1 \times 10^{5} 4 \mathrm{~T} 1$ cells were orthotopically inoculated at the forth mammary fat pad of 
6-8-week-old female BALB/c mice. Tumor volumes were monitored every 2 days after inoculation. When the average sizes got to about $100 \mathrm{~mm}^{3}$, mice bearing 4T1 tumor were allocated into five groups (saline, M-PLL/siRNA ${ }^{\text {N.C. }}$, M-PLL/siRNA ${ }^{\text {N.C.+NIR, M-PLL/siRNA }}{ }^{\text {Sur }}$, and M-PLL/ siRNA ${ }^{\text {Sur }}+$ NIR) at random and intratumorally injected at a dosage of $0.3 \mathrm{mg}$ siRNA $\mathrm{kg}^{-1}$, respectively. At $3 \mathrm{~h}$ after injection, tumors were irradiated by NIR laser at $808 \mathrm{~nm}$ and $1.5 \mathrm{~W} \mathrm{~cm}^{-2}$ for $180 \mathrm{~s}$. The treatment was administrated every other day for total five times. On the third day after final treatment, mice were sacrificed. Tissues of lungs and tumors were excised. To observe the metastatic nodules, lung tissues were immersed in Bouin's solution for $16 \mathrm{~h}$ and photographed. Then, tumors and lungs were fixed in $4 \%(\mathrm{w} / \mathrm{v})$ paraformaldehyde solution, embedded into paraffin and examined with hematoxylin and eosin ( $\mathrm{H} \& \mathrm{E})$ staining method to evaluate the antimetastatic efficacy of M-PLL-NPs.

\section{Statistical analyses}

The data were presented with mean and SD. All statistical analyses were performed using SPSS 22.0 for Windows (IBM Corporation, Armonk, NY, USA). Statistical comparisons were performed to determine group differences through analysis of variance. $P<0.05$ was viewed as statistically significant.

\section{Results and discussion}

Successful escape from lysosomes is necessary for siRNA to implement gene silencing before lysosome-mediated digestion. Recently, NIR irradiation-induced lysosomal escape of siRNA has gained enormous attentions through photothermal destruction of lysosomal membrane. ${ }^{9,14-16}$ As an endogenous photothermal sensitizer, melanin has been engineered as a polymer backbone for efficiently delivering drugs due to its good biocompatibility and biodegradability. ${ }^{12,25}$ In this study, we constructed a melanin-based nanoparticle as a low toxic and potent tool for nucleic acid delivery. Taking advantage of the deep penetration of NIR light and efficient photothermal conversion of melanin, the NIR light induced local heat that could accelerate endosomal escape of siRNA for enhancing gene silencing. Furthermore, since NIR could be spatiotemporally controlled, therapeutic RNAi might profit from this tactic, whereas avoiding unnecessary adverse effects.

\section{Preparation and characterization of M-PLL/siRNA nanoparticles}

First, the copolymer of M-PLL was synthesized according to a well-developed method. ${ }^{23}$ The PLL was conjugated with melanin through a reaction between the amine groups in
PLL molecules and dihydroxyindole/indolequinone groups of melanin (Figure 1A). To confirm successful conjugation, the structural composition of M-PLL copolymer was characterized by FT-IR spectra and ${ }^{1} \mathrm{H}-\mathrm{NMR}$. As depicted in Figure 1B, the absorption peak at 3,000-3,500 $\mathrm{cm}^{-1}$ belonged to $v_{\mathrm{NH}}$ stretch vibration, and the characteristic peaks at $1,657 \mathrm{~cm}^{-1}$ and $1,530 \mathrm{~cm}^{-1}$ represented the amide I bond and amide II bond in the PLL, respectively. Figure 1C provides the ${ }^{1} \mathrm{H}-\mathrm{NMR}$ spectra of MNP and M-PLL in $\mathrm{D}_{2} \mathrm{O}$. As described in the literature, there was no obvious signal of the hydrogen atom on the arylene groups in the ${ }^{1} \mathrm{H}-\mathrm{NMR}$ spectrum of MNP (Figure 1Ca) because most of the conjugated backbones were buried. ${ }^{23}$ The new characteristic peaks $\left[\left(\delta\left(\mathrm{CH}_{2}\right)_{3}\right)=1.20-1.81,\left(\delta-\mathrm{CH}_{2}\right)=2.92,(\delta(-\mathrm{HN}-\mathrm{CH}<))=4.23\right]$ were attributed to the conjugated PLL (Figure 1Cb), which verified the presence of PLL on the MNP. Moreover, the introduction of PLL significantly improved water solubility of primitive melanin particle as expected (Figure 1D). Then, the positively charged M-PLL complexed with negatively charged siRNA through electrostatic interaction to form polyplexes. Electrostatic-binding capacity between M-PLL and siRNA was verified by gel electrophoresis test at various weight ratios (polymer/siRNA). As shown in Figure 1E, M-PLL could completely condense siRNA over the weight ratio of $5: 1$. Therefore, we optimized the weight ratio by investigating the silencing luciferase expression ability of M-PLL/siRNA ${ }^{\text {Luc }}$ at weight ratios 5:1, 10:1, 20:1, 40:1, and 60:1 without NIR light (data were not shown). As a result, the weight ratio of 40 was optimized and chosen for in vitro and in vivo studies in this work. Figure $1 \mathrm{G}$ displays the representative image of M-PLL/siRNA nanoparticles with flower-like shape and uniform size distribution. The hydrodynamic size determined by DLS was about $47.65 \mathrm{~nm}$ (Figure 1F), and the zeta potential was $+7.23 \mathrm{mV}$.

\section{The photothermal property of M-PLL in vitro}

Melanin could produce heat under NIR light due to their strong absorption in both far- and NIR bands. To study the heat generation capability, M-PLL was examined under NIR irradiation $\left(1.5 \mathrm{~W} \mathrm{~cm}^{-2}\right)$ by using the PBS and melanin as controls. M-PLL exhibited intensive absorbance over a wide range (Figure 2A) and allowed fast heat generation under $808 \mathrm{~nm}$ NIR laser irradiation (Figure 2B). After continuous NIR irradiation for $5 \mathrm{~min}$, the temperature of melanin and M-PLL both dramatically raised from $21.7^{\circ} \mathrm{C}$ to above $50^{\circ} \mathrm{C}$, whereas the PBS solution only heated up to $23.8^{\circ} \mathrm{C} \pm 0.5^{\circ} \mathrm{C}$. This result indicated that compared with melanin, the attachment of PLL polymer did not affect M-PLL as a 
A

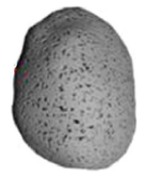

$\underset{\begin{array}{c}\text { 2. Neutralized } \\ \text { under sonication }\end{array}}{\stackrel{0.1 \mathrm{M} \mathrm{NaOH}}{\text { noH }}}$

Melanin granule

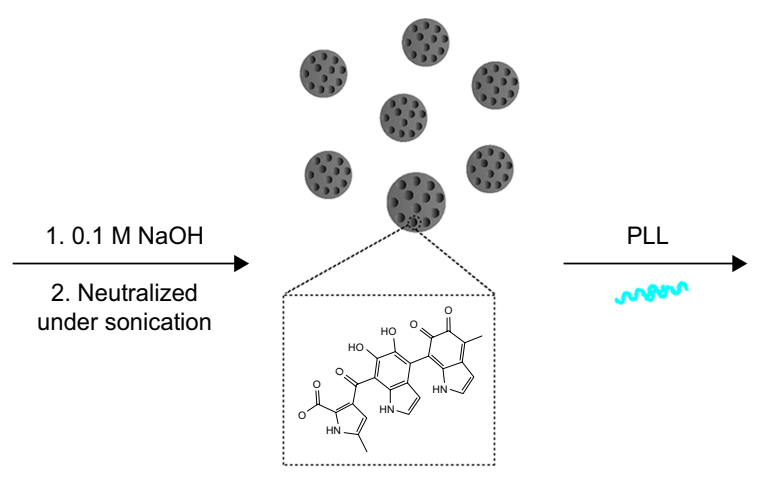

MNP

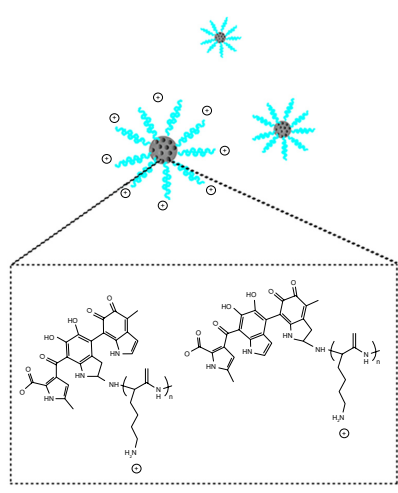

M-PLL
B
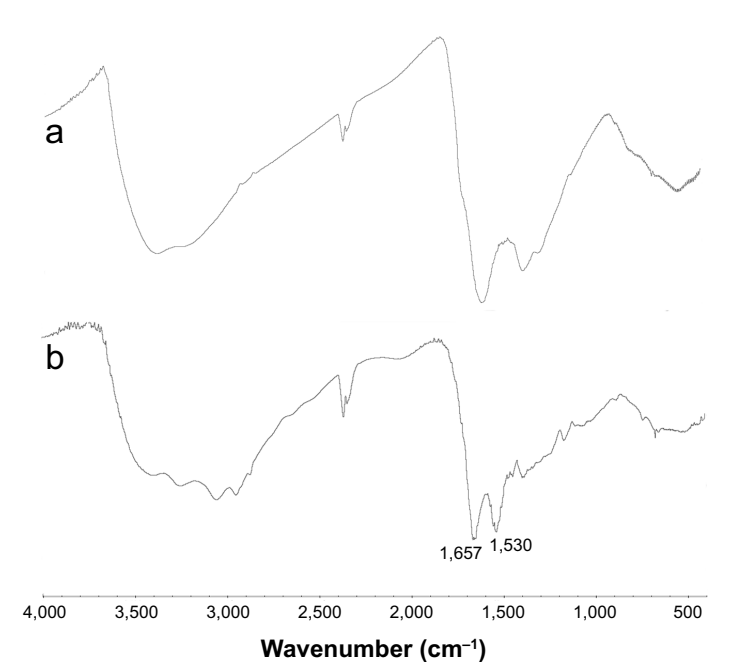

C
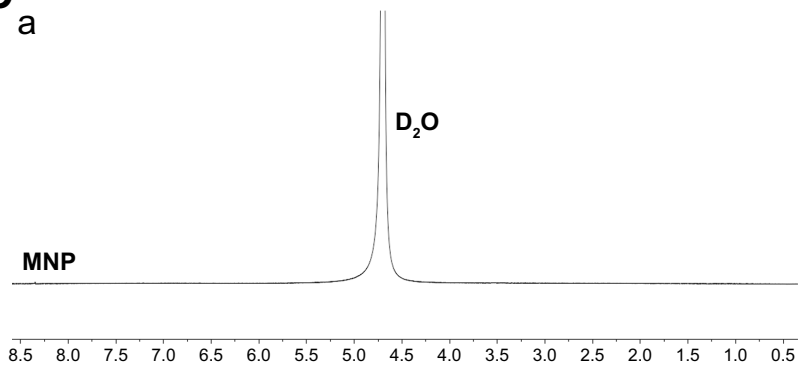

f1 (ppm)

b

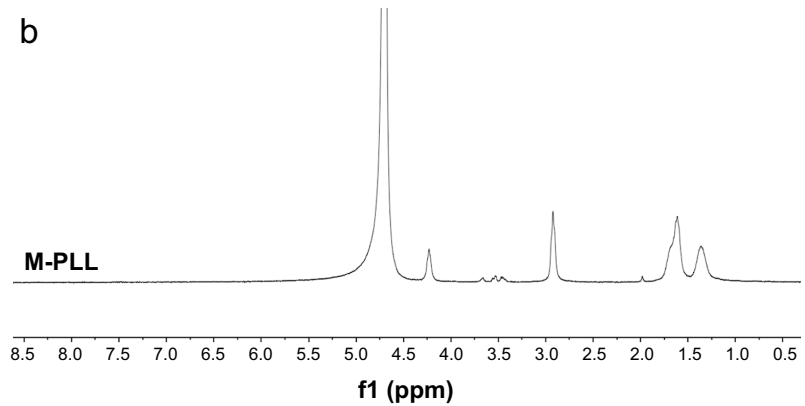

D

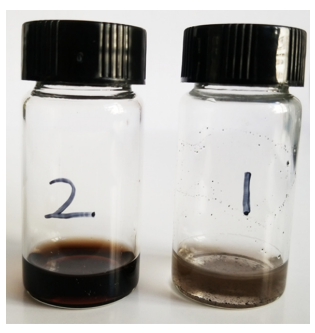

E
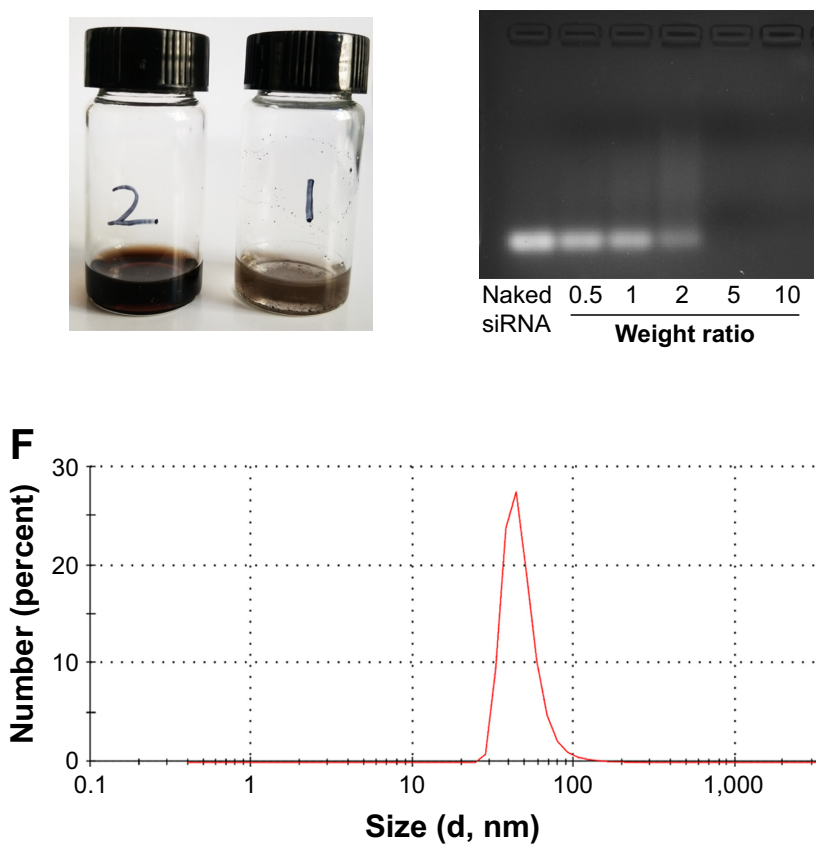

G

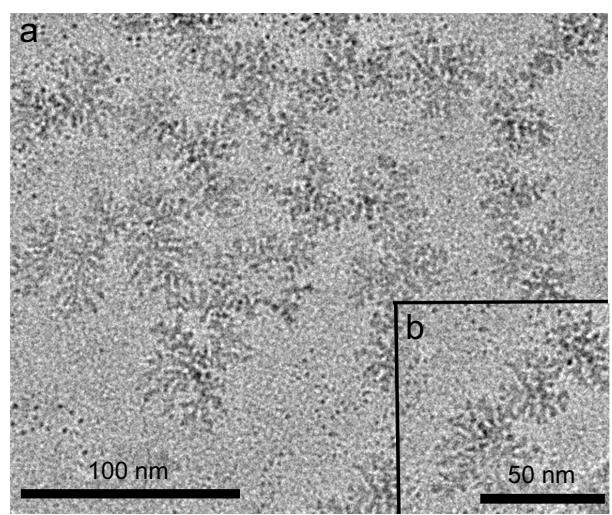

Figure I Synthesis and characterization of M-PLL.

Notes: (A) Synthetic scheme of M-PLL. (B) FT-IR spectra of MNP (a) and M-PLL (b). (C) 'H-NMR spectra of MNP (a) and M-PLL (b) in D 2 O. (D) Pictures of M-PLL (left) and melanin (right) in $\mathrm{H}_{2} \mathrm{O}$. (E) Gel retardation assay with M-PLL/siRNA polyplexes at various weight ratios. (F) Size distribution and (G) TEM image of M-PLL-NPs prepared at a weight ratio of 40 . The scale bar represented $100 \mathrm{~nm}$ (a) and $50 \mathrm{~nm}$ (b). Magnification $\times 100,000$.

Abbreviations: FT-IR, Fourier transform-infrared; M-PLL, melanin-poly-L-lysine; M-PLL-NPs, melanin-poly-L-lysine/siRNA nanoparticles; MNP, melanin nanoparticle; PLL, poly-L-lysine; siRNA, small interference RNA; TEM, transmission electron microscopy. 
A

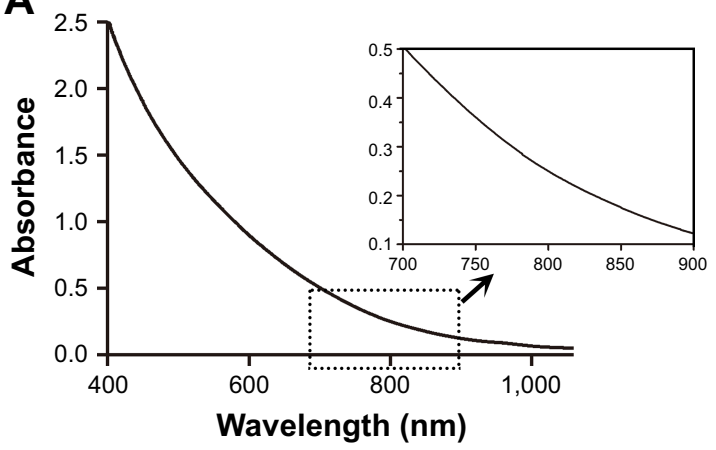

B

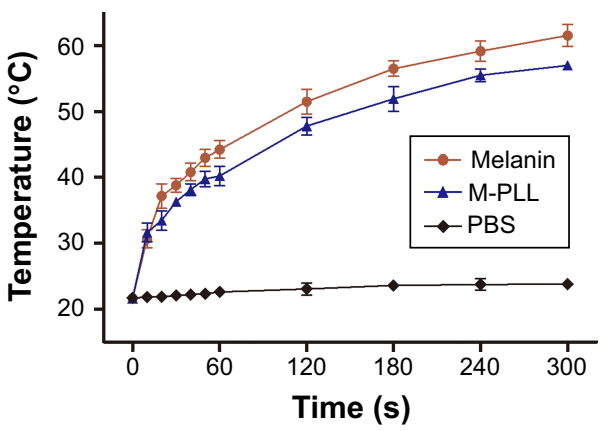

C

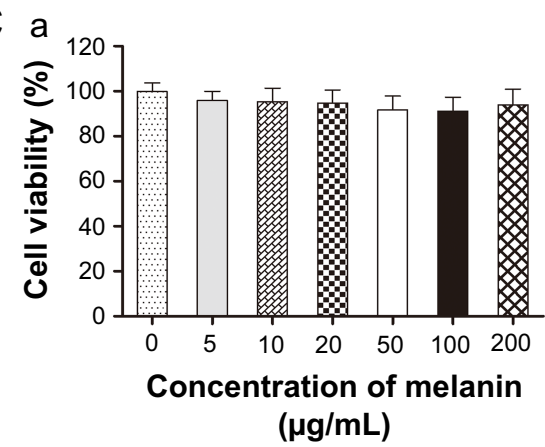

b

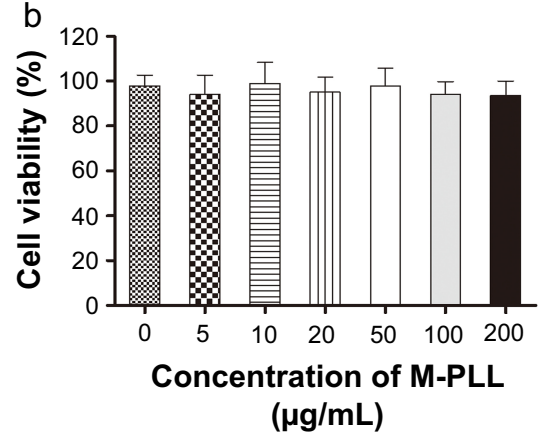

D

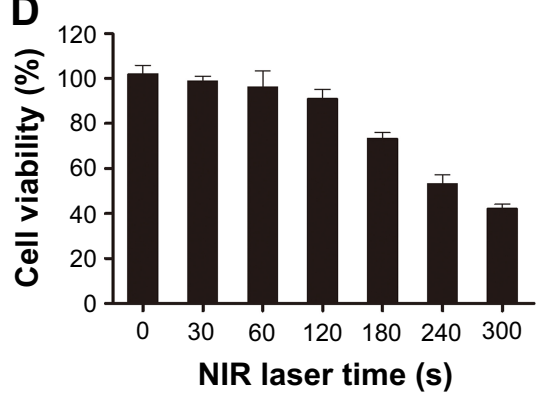

Figure 2 (A) Absorbance spectra of M-PLL. (B) Photothermal effect of melanin and M-PLL with NIR irradiation at $808 \mathrm{~nm}$ and the power density of I.5 W $\mathrm{cm}{ }^{-2}$ ( $\mathrm{n}=3$, mean $\pm S D)$. (C) Cytotoxicity of melanin (a) and M-PLL (b) on 4TI cells ( $n=6$, mean $\pm S D)$. (D) Cell viability profile of M-PLL/siRNA ${ }^{N . C}$ nanoparticles after NIR irradiation on $4 \mathrm{TI}$ cells at a power density of $1.5 \mathrm{~W} \mathrm{~cm}^{-2}$ at different seconds ( $n=6$, mean $\pm \mathrm{SD}$ ). siRNAs of nonsense sequences.

Abbreviations: M-PLL, melanin-poly-L-lysine; siRNA ${ }^{\text {N.C. }}$, siRNA of nonsense sequences; NIR, near infrared; PBS, phosphate-buffered solution.

preferable candidate for effective light-thermal conversion. The superior photothermal effect of M-PLL can make it useful in PTT-controllable therapeutic applications.

\section{Cytotoxicity of M-PLL}

The cytotoxic influence of M-PLL copolymer was studied by CCK 8 assay on 4T1 cells. After incubation for $24 \mathrm{~h}$, both melanin and the polymer M-PLL showed no obvious cytotoxicity at concentrations of $0-200 \mu \mathrm{g} \mathrm{mL}-1$ (Figure 2C), which suggested that M-PLL copolymer could be a secure non-viral vector in this concentration range. Moreover, the cytotoxicity of M-PLL-NPs (the polyplexes of M-PLL and siRNA ${ }^{\text {N.C. }}$ ) under NIR irradiation was also studied to evaluate the potential influence of laser. As displayed in Figure 2D, the viability of 4T1 cells decreased with increasing exposure time. When the output power of $1.5 \mathrm{~W} \mathrm{~cm}^{-2}$ was fixed below $120 \mathrm{~s}$, the cell viability was maintained above $90 \%$. However, the luciferase expression induced by M-PLL/siRNA ${ }^{\text {Luc }}+$ NIR was as the same level as M-PLL/siRNA ${ }^{\text {Luc }}$ without NIR light (data were not shown). As the exposure time extended to $180 \mathrm{~s}$, the luciferase expression of cells in M-PLL/siRNA ${ }^{\text {Luc }}+\mathrm{NIR}$ group significantly decreased (Figure 3B), with cell survival rate of about $73.4 \%$ (Figure 2D). At exposure time more than $180 \mathrm{~s}$, significantly more cells were killed, probably due to photothermal ablation mediated by M-PLL inside cells. Taking into consideration of the photothermal property and cytotoxic effect of M-PLL, $1.5 \mathrm{~W} \mathrm{~cm}^{-2}$ for $180 \mathrm{~s}$ was chosen as a ceiling dose in the following gene silencing experiments.

\section{Photothermally triggered enhanced endosome escape and downregulation of endogenous luciferase gene expression}

Generally, after entering cells via the endocytic pathway, the polyplexes formed by cationic vectors and siRNA will be entrapped in early endosomes, which would rapidly maturate into late endosomes and ultimately fuse with other late endosomes or lysosomes. ${ }^{26,27}$ Therefore, effective escape from late endosomes or lysosomes is a critical step for siRNA to achieve gene silencing before digestion in lysosome. To demonstrate the enhanced endosomal escape by NIR-activated photothermal effect, we observed the intracellular fate of M-PLL-NPs after NIR irradiation. Herein, LysoTracker Red, a marker with red fluorescence, was used to label the late endosomes and lysosomes in 4T1 cells. As displayed in confocal images, without NIR treatment, bright yellow fluorescence was observed in Figure 3Aa, 

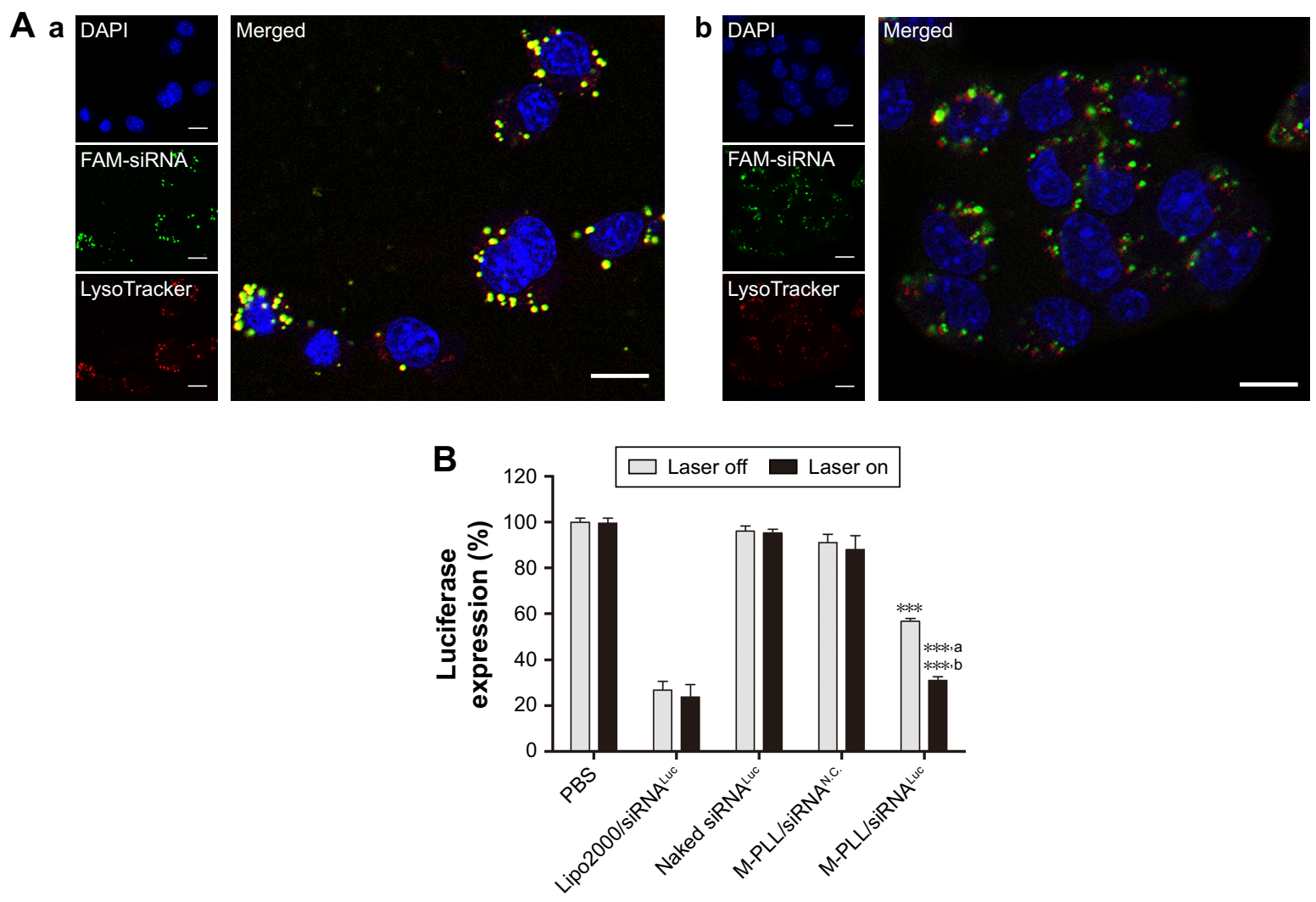

Figure 3 (A) CLSM images of 4TI cells treated with M-PLL/FAM-siRNA nanoparticles in the dark (a) or under NIR irradiation (b). The DAPI (blue) and LysoTracker ${ }^{\circledR}$ Red DND-99 (red) were used for staining nucleus and endosomes/lysosomes, respectively. Scale bar is $10 \mu$ m, magnification $\times 400$. (B) Relative luciferase expression of 4 TI Luc cells after treated with M-PLL/siRNA ${ }^{\text {Luc }}$ nanoparticles $(n=3$, mean $\pm S D)$. $* * * P<0.0$ I by comparing M-PLL/siRNA ${ }^{\text {Luc }}$ (Laser off) with PBS, naked siRNA ${ }^{\text {Luc }}$ and M-PLL/siRNA ${ }^{\text {N.C. }}$, $* * *, a \mathrm{P}<0.00$ I by comparing M-PLL/siRNA ${ }^{\text {Luc }}$ (Laser on) with PBS, naked siRNA ${ }^{\text {Luc }}$ and M-PLL/siRNA ${ }^{\mathrm{N} . c \text {. }}$ and $* * *, b \mathrm{P}<0.00 \mathrm{I}$ by comparing M-PLL/siRNA ${ }^{\text {Luc }}$ (Laser on) with M-PLL/ siRNA ${ }^{\text {Luc }}$ (Laser off).

Abbreviations: CLSM, confocal laser scanning microscopy; luc, luciferase; siRNA ${ }_{\text {Luc }}$ luciferase-targeted siRNA; M-PLL, melanin-poly-L-lysine; siRNAN.C., siRNA of nonsense sequences; NIR, near infrared; PBS, phosphate-buffered solution; siRNA, small interference RNA; siRNA ${ }^{\text {sur }}$, survivin-targeted siRNA.

which was the overlapped signal of FAM-siRNA (green) and endosome/lysosome (red). On the contrary, after cells treated with NIR laser, the green fluorescence was separated with the red region (Figure 3Ab). These phenomena indicated an accelerated endosomal escape of siRNA induced by NIR irradiation, which was well in accordance with our hypothesis that "the photothermal effect" of melanin could disrupt the endosomal/lysosomal membrane and facilitate siRNA delivery into cytoplasm. Consequently, we assumed that enhanced gene silencing of target cells might be achieved with the help of NIR irradiation. Thus, the luciferase gene expression assay was subsequently evaluated with $4 \mathrm{~T} 1^{\mathrm{Luc}}$ cells, which was stably expressing the firefly luciferase, to explore the potential of M-PLL as a preferable siRNA vector. As presented in Figure 3B, whether the laser was on or off, the naked siRNA ${ }^{\text {Luc }}$ showed negligible transfection ability to silence luciferase expression, while Lipofectamine 2000/ siRNA ${ }^{\text {Luc }}$ presented about $20 \%$ luciferase expression, which is acknowledged as an efficient and commercial transfection reagent in many cell types. But the case was quite different as to M-PLL-NPs. In the M-PLL/siRNA ${ }^{\text {Luc }}$ group, the luciferase activity was about $56.75 \% \pm 1.28 \%$ without light, whereas the luciferase expression decreased to $31.03 \% \pm 1.61 \%$ under the stimulus of light. The luciferase downregulation ability of M M-PLL/siRNA ${ }^{\text {Luc }}$ under NIR illumination was about 1.82 times higher than that without NIR $(P<0.001)$. From all these results, we can conclude that the light-generated heat effect of melanin facilitated M-PLL-NPs to accelerate escape rate from endosomes, which resulted in enhancement of gene silencing activity.

\section{In vitro anti-proliferation and apoptotic effect of photothermal-inducible survivin- targeted gene silencing}

To investigate the therapeutic efficacy of photothermalinducible M-PLL/siRNA nanoparticles, the cell proliferation-inhibiting effect of different formulations was first tested by CCK 8 assay in 4T1 cells. Herein, survivin was used as the target gene to be silenced due to its vital role in the breast cancer progression and metastasis.$^{28}$ Cell viability 
in PBS and naked siRNA group remained above $90 \%$ regardless of NIR irradiation (Figure 4A). However, the viability of M-PLL/siRNA ${ }^{\text {Sur }}$-treated cells declined about $30 \%$ after exposed to NIR laser. When compared with the dark condition, M-PLL/siRNA ${ }^{\text {Sur }}$ exhibited significant inhibitory effect in the NIR condition, which undoubtedly indicated the enhanced gene silencing efficacy due to the light-generated heat effect.

Then, Live/Dead cytotoxicity assay was performed to evaluate the inhibitory effect on 4T1 cells by using calcein-AM/PI agents (Figure 4B). Live cells were stained by calcein-AM and generated bright green fluorescence; nevertheless, dead cells showed red fluorescence as a result of staining by PI. In contrast to other groups, the number of dead cells significantly increased when treated with M-PLL/ siRNA ${ }^{\text {Sur }}$ with or without NIR laser. Furthermore, more red fluorescence could be observed in M-PLL-siRNA ${ }^{\text {Sur }}+$ NIR group when compared with M-PLL-siRNA ${ }^{\text {Sur }}$, which indicated that more dead cells were induced after NIR irradiation exposure. These results were in accordance with the abovementioned CCK8 test results, which proved a synergistic effect to facilitate endosomal escape for improved intracellular delivery of siRNA and gene silencing.

To elucidate photothermally induced apoptosis rather than necrosis that led to effective anti-proliferation, we performed cell apoptosis experiments. The apoptosis rate of 4T1 cells was quantitatively detected by Annexin V-FITC/PI double-staining assay. As displayed in Figure 4C, after $24 \mathrm{~h}$ treatment, the total ratio of apoptosis in M-PLL/ siRNA ${ }^{\text {Sur }}+$ NIR group was $48.53 \%$; nevertheless, the percentages in PBS, naked siRNA ${ }^{\text {Sur }}$, and M-PLL/siRNA ${ }^{\text {Sur }}$ groups were $3.51 \%, 4.78 \%$, and $22.91 \%$, respectively. Moreover, compared with PBS, cells in M-PLL/siRNA ${ }^{\text {N.C.+NIR group }}$ exhibited only a slightly higher apoptosis (6.48\%). These data manifested that the significantly inhibitory and apoptotic effect of M-PLL/siRNA ${ }^{\text {Sur }}+$ NIR was mainly owing to enhanced gene silencing effect of survivin-targeted siRNA assisted by NIR irradiation.

\section{Survivin downregulation in vitro}

The results of the ELISA assay shown in Figure 4D revealed that compared with PBS and naked siRNA ${ }^{\text {Sur }}$ treatment, M-PLL/siRNA ${ }^{\text {Sur }}$ induced more downregulation of survivin protein regardless of NIR irradiation. Moreover, the amount of survivin protein ranged from $4.11 \pm 0.59 \mathrm{ng} \mathrm{mg}^{-1}$ to $1.63 \pm 0.37 \mathrm{ng} \mathrm{mg}^{-1}$ after NIR light treatment, which indicated an improved ability of M-PLL/siRNA ${ }^{\text {Sur }}$ for silencing target gene with the aid of efficient photothermal transfection.

\section{Inhibition of tumor growth and metastasis in vivo}

As the mean tumor volume was $\sim 100 \mathrm{~mm}^{3}$, mice were distributed into five groups in accordance with the principle of randomization and intratumorally injected with saline, M-PLL/siRNA ${ }^{\text {N.C. }}$, M-PLL/siRNA ${ }^{\text {N.C. }}+$ NIR, M-PLL/ siRNA $^{\text {Sur }}$, and M-PLL/siRNA ${ }^{\text {Sur }}+$ NIR. It can be observed in Figure 5A that after treatments, the tumor volume in both saline group and M-PLL/siRNA ${ }^{\text {N.C. }}$ group grew up fast $(P>0.05)$, which implied that without NIR irradiation the polymer of M-PLL itself had no inhibitory effect on 4T1 tumor. The tumor growth rate in M-PLL/siRNA ${ }^{\text {N.C. }}+\mathrm{NIR}$ group slightly slowed down; however, there was no significant difference when compared with saline treatment $(P<0.05)$. Obviously, the tumors of mice in both siRNA ${ }^{\text {Sur }}$ loaded M-PLL nanoparticles groups grew very slowly no matter whether NIR irradiated tumors or not. At the end of treatment, the tumor volumes in both M-PLL/siRNA ${ }^{\text {Sur }}$ and M-PLL/siRNA ${ }^{\text {Sur }+N I R ~ g r o u p s ~ w e r e ~ s i g n i f i c a n t l y ~ s m a l l e r ~ t h a n ~}$ that in the saline group $(P<0.001)$. Furthermore, compared to the M-PLL/siRNA ${ }^{\text {Sur }}$, tumors in M-PLL/siRNA ${ }^{\text {Sur }}+\mathrm{NIR}$ group grew more slowly and the tumor volumes displayed significant differences at the end of therapy $(P<0.05)$. During the period of treatment, no obvious body weight varied for each group of mice (Figure 5B), indicating no fearful toxicity of M-PLL NPs.

It was well known that 4T1 cells were highly malignant and metastasizing. When inoculated orthotopically, 4T1 tumor could metastasize to the lungs. ${ }^{29,30}$ Hence, at the end of treatment, lungs were isolated from sacrificed mice to further evaluate antitumor effect of M-PLL-NPs. First, lung metastatic foci were observed and photographed after lungs were immersed in Bouin's fixative. The number and scale of white nodules indicated the metastatic colonization of 4T1 cells in lungs. In the obtained photographs (Figure 5C), distinct metastatic nodules could be observed in the groups of saline, M-PLL/siRNA ${ }^{\text {N.C., and M-PLL/siRNA }}{ }^{\text {N.C. }}+$ NIR. But the metastatic colonization was obviously lessened in M-PLL/siRNA ${ }^{\text {Sur }}$ treating group. Notably, no visible nodules could be observed in the lungs of M-PLL/siRNA ${ }^{\text {Sur }}+$ NIR group, implying that mice might be free from lung metastasis. In order to get further verification, fixed lung tissues in all groups are stained with H\&E for histopathological examination, and the representative images are displayed in Figure 5D. In accordance with the results in Figure 5C, big metastatic focus could be observed in pathological slices of lung tissues of mice from saline, M-PLL/siRNA ${ }^{\text {N.C. }}$, and M-PLL/siRNA ${ }^{\text {N.C. }}+$ NIR groups, whereas only small 

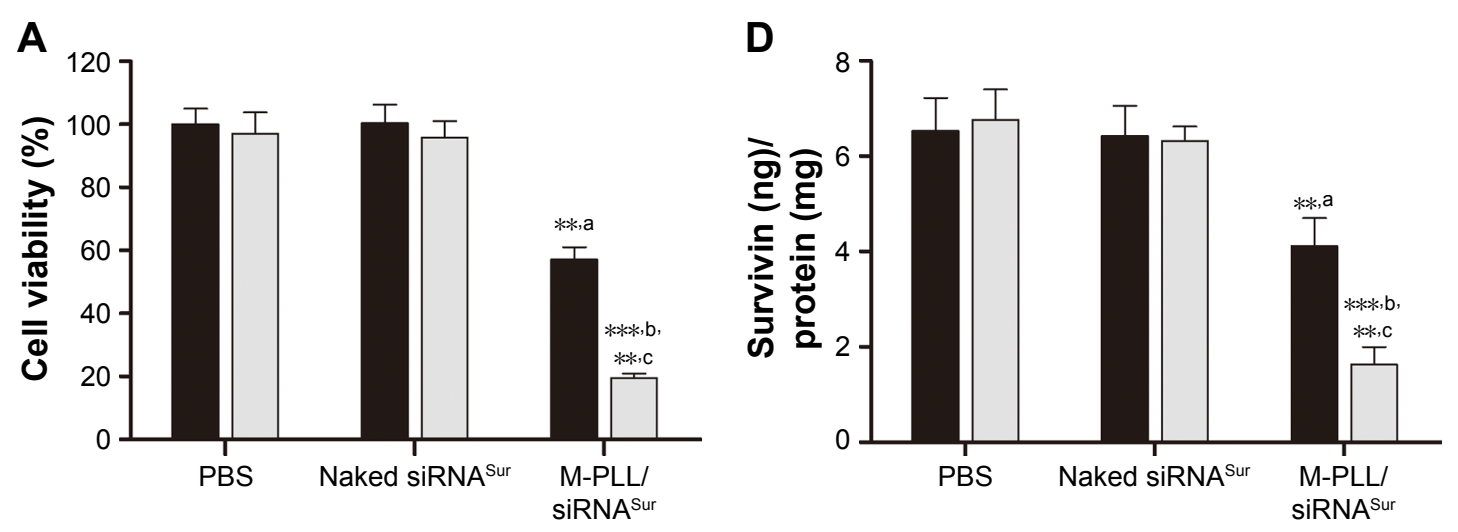

Laser off $\square$ Laser on

B

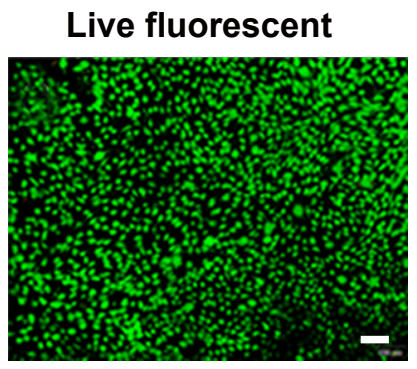

Dead fluorescent
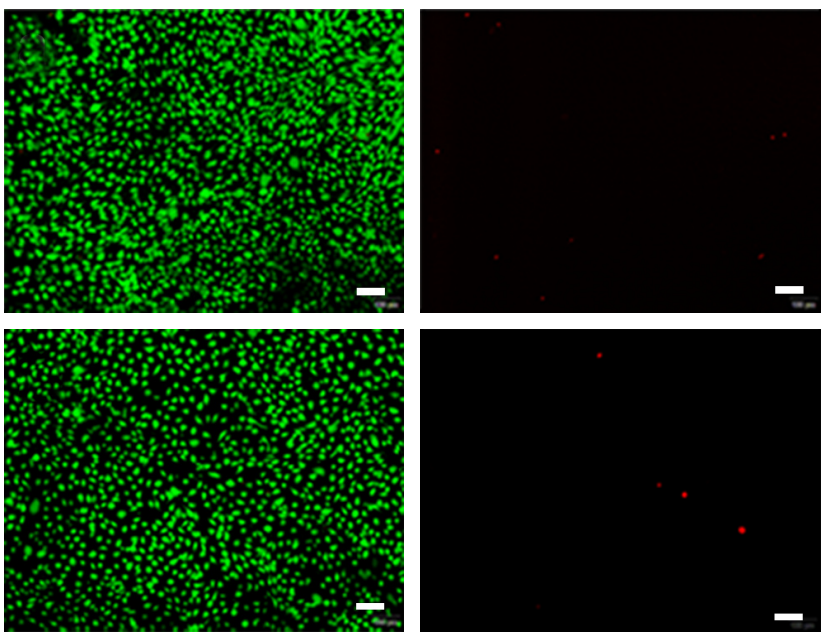

\section{Live/dead merged}
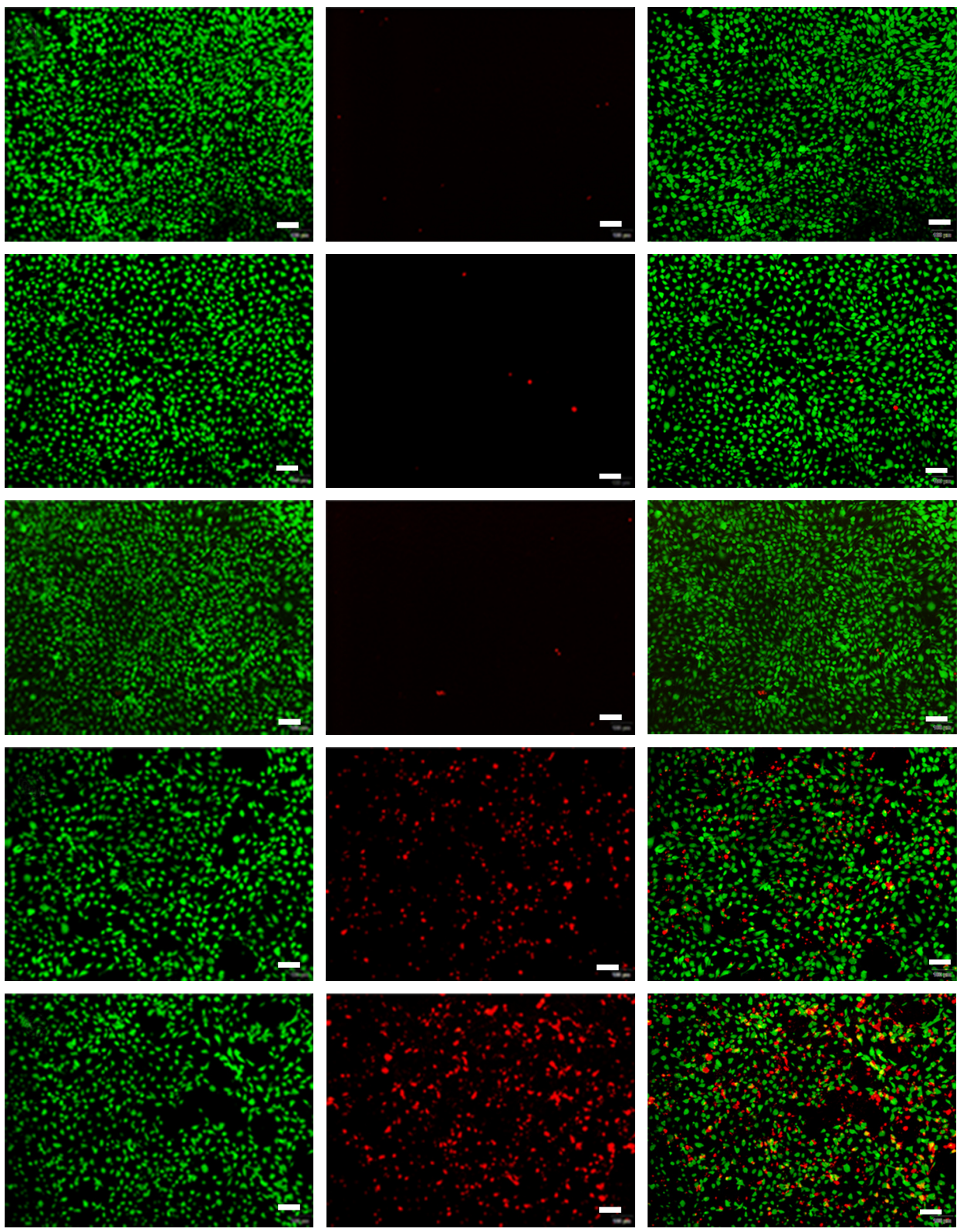

Figure 4 (Continued) 

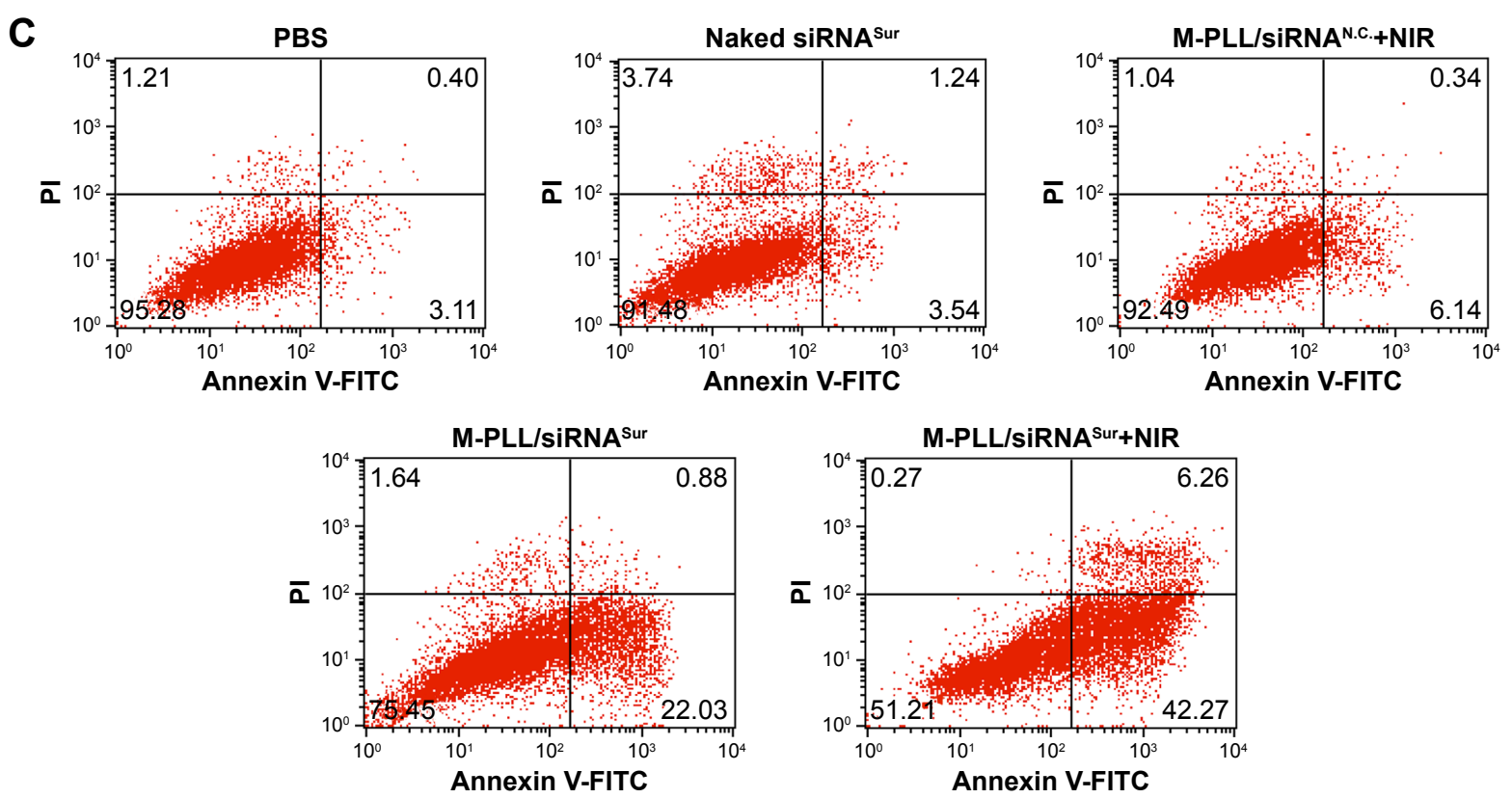

Figure 4 (A) Proliferation inhibition assay of M-PLL/siRNA ${ }^{\text {sur }}$ nanoparticles on 4TI cells with or without NIR irradiation ( $n=6$, mean \pm SD). (B) Representative cytotoxicity image of 4TI cells incubated with PBS (a), naked siRNA ${ }^{\text {sur }}$ (b), M-PLL/siRNA ${ }^{\text {N.c. }}$ (c), M-PLL/siRNA ${ }^{\text {sur }}$ (d) or M-PLL/siRNA ${ }^{\text {Sur }}+N I R$ (e) by using calcein-AM (green, live cells) and propidium iodide (red, dead cells) staining method. Scale bar is $100 \mu \mathrm{m}$, magnification $\times 40$. (C) Quantitatively apoptotic analysis of cells treated with different formulations by using Annexin V-FITC/PI staining assay. (D) Survivin protein levels in 4TI cells after different treatment by ELISA method. Data were presented as nanogram of surviving protein per milligram of protein $\left(n=3\right.$, mean $\pm S D$ ). $* *, a P<0.0$ I by comparing M-PLL/siRNA ${ }^{\text {sur }}$ (Laser off) with PBS and naked siRNA ${ }^{\text {Sur, }} * * *, b P<0.00$ I by comparing $M-P L L /$ siRNA $^{\text {sur }}$ (Laser on) with PBS and naked siRNA ${ }^{\text {sur }}$ and $* *, c P<0.01$ by comparing M-PLL/siRNA ${ }^{\text {sur }}$ (Laser on) with M-PLL/siRNA ${ }^{\text {sur }}$ (Laser off).

Abbreviations: M-PLL, melanin-poly-L-lysine; NIR, near infrared; siRNA, small interference RNA; PBS, phosphate-buffered solution; siRNA ${ }^{\text {N.C. }}$, siRNA of nonsense sequences;

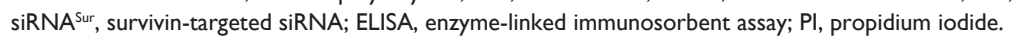

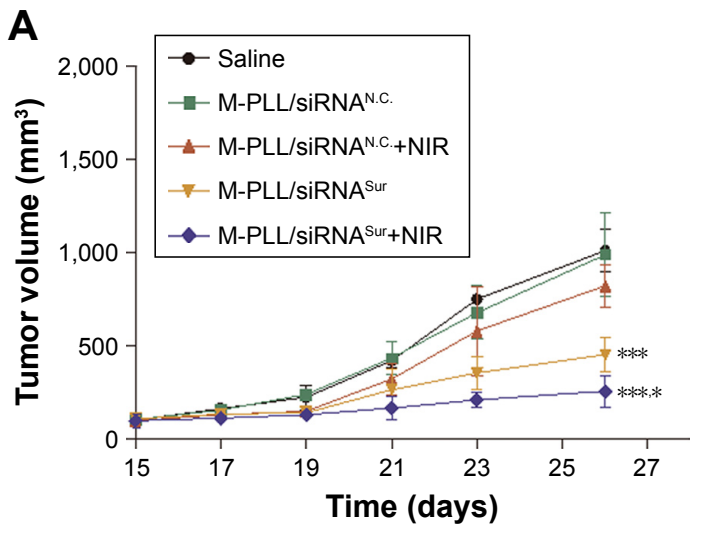

C

a

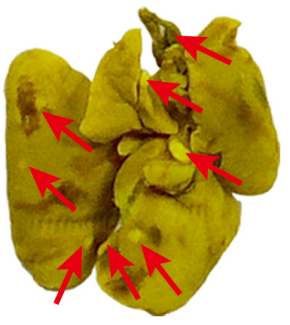

b

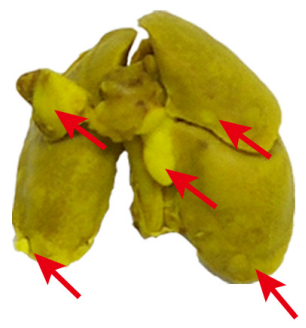

e

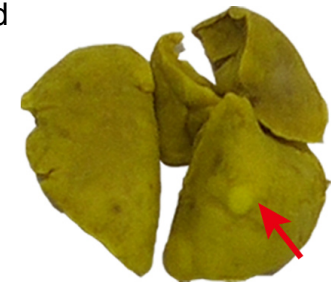

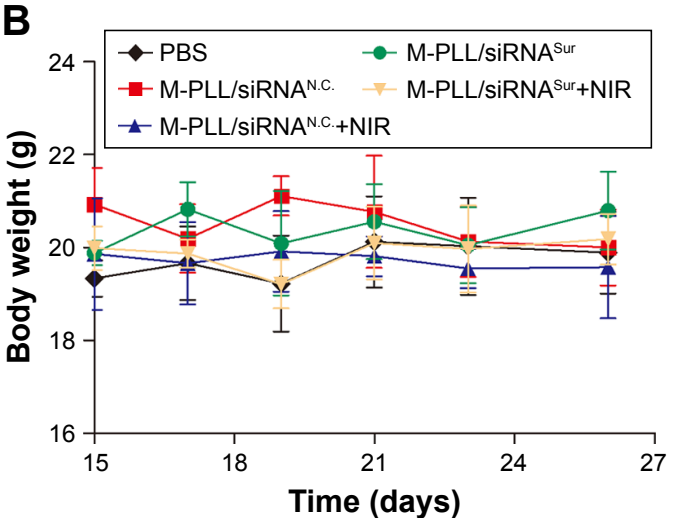

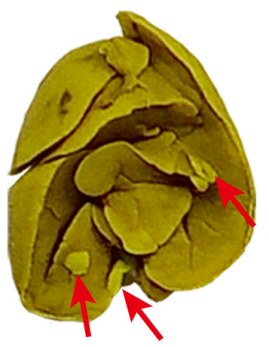

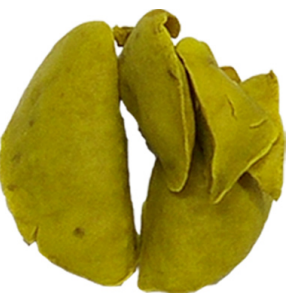



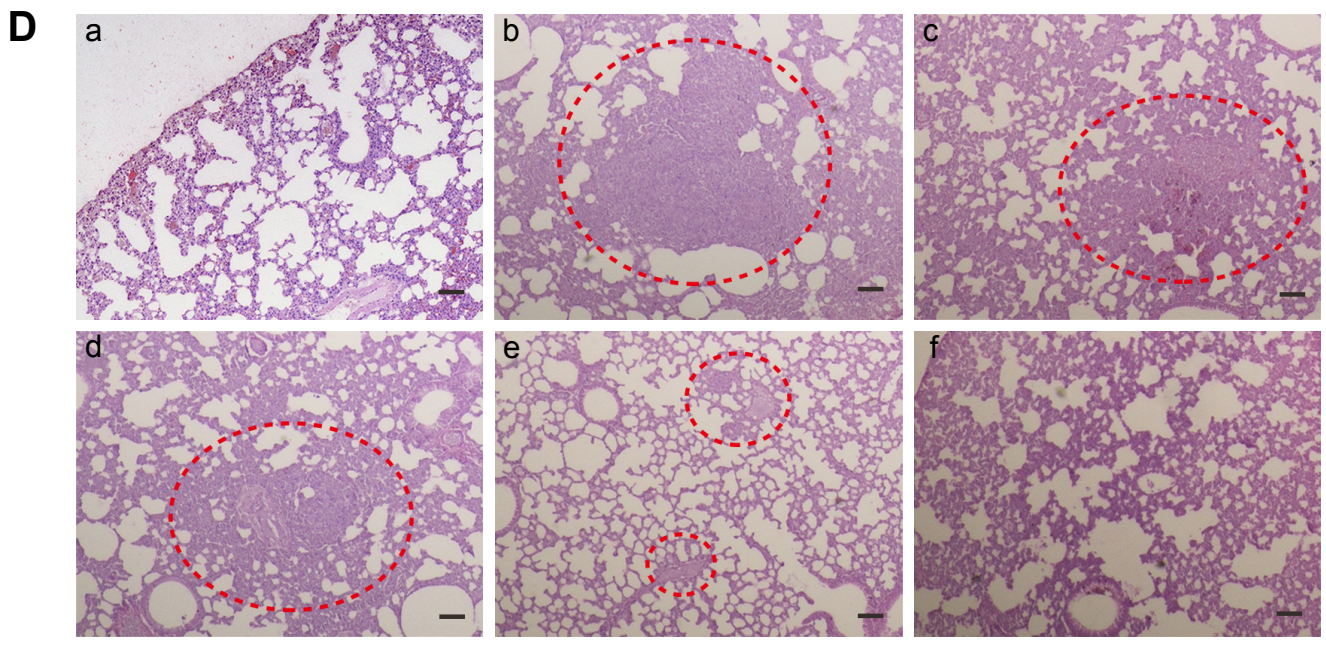

Figure 5 In vivo antitumor effect on 4TI tumor-bearing Balb/c mice.

Notes: (A) Tumor volume of mice treated with different formulations $(n=3)$. $* * * P<0.00$ I represents M-PLL/siRNA ${ }^{\text {Sur }}$ group and M-PLL/siRNA ${ }^{\text {Sur }}+N I R$ group versus the saline group, respectively; $* P<0.05$ represents $M-P L L / s i R N A^{\text {Sur }}+N I R$ group versus the M-PLL/siRNA ${ }^{\text {sur }}$ group. (B) Body weight variation of mice in the duration of treatments. (C) Typical lung tissue photographs of $4 T$ I tumor-bearing mice treated with saline (a), M-PLL/siRNA ${ }^{N . C .}$ (b), M-PLL/siRNA ${ }^{N . C}+{ }^{+N I R}$ (c), M-PLL/siRNA ${ }^{\text {Sur }}$ (d) or M-PLL/ siRNA $^{\text {Sur }}+$ NIR (e), respectively. Red arrows indicate the white nodules on the surfaces of lung lobes. (D) Representative H\&E-stained lung section of 4TI tumor-bearing mice

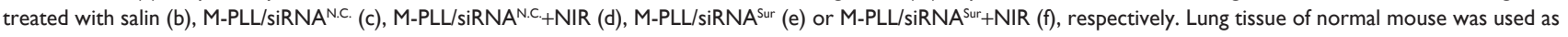
a control (a). Red dotted circles show the tumor metastasis loci in the lungs. Scale bar is $100 \mu \mathrm{m}$, magnification $\times 40$.

Abbreviations: H\&E, hematoxylin and eosin; M-PLL, melanin-poly-L-lysine; siRNAN.C., siRNA of nonsense sequences; NIR, near infrared; PBS, phosphate-buffered solution; siRNA, small interference RNA; siRNA ${ }^{\text {sur }}$, survivin-targeted siRNA.

metastatic foci were found in the lung specimen of mice from M-PLL/siRNA ${ }^{\text {Sur }}$ group. Additionally, clear structure and alveolar septum could be observed in the lung pathological section of mice from M-PLL/siRNA ${ }^{\text {Sur }}+$ NIR group, which was similar with that from normal mice. These results not only confirmed the therapeutic effect of M-PLL/siRNA ${ }^{\text {Sur }}$ in inhibiting 4T1 tumor growth and metastasis in vivo but also demonstrated that photothermally triggered cytosolic siRNA delivery had the potential for improving RNAi therapy.

\section{Conclusion}

We have successfully fabricated a good biocompatible nanosystem based on M-PLL polymer for siRNA delivery. Due to the excellent photothermal effect of melanin, the M-PLLNPs could overcome endosomal barrier and effectively release siRNA into cytoplasm under the NIR stimulus. As a result, the M-PLL/siRNA nanoparticle achieved efficient gene silencing capacity and exhibited great antitumor effect on 4T1 breast tumor model. These findings demonstrated that the M-PLL-NPs could be a promising non-viral vector for siRNA delivery and possess the potential for enhanced RNAi therapy.

\section{Acknowledgments}

This work was financially supported by the National Natural Science Foundation of China (grant nos 81571747 and 81771907), Fund Program for the Scientific Activities of Selected Returned Overseas Professionals in Shanxi Province,
Shanxi Scholarship Council of China (no 2015057), Key research and development project of Shanxi Province (nos 2016021 and 201703D321015-3), Science and technology innovation team project (no 201705D131026), Scientific and technological achievements transformation project of Shanxi Province (no 201704D131006), Shanxi Province Science Foundation for Youths (no 201701D221257), and the Foundation for PhD of Shanxi Medical University (no 03201620). Xi Yang, Bo Fan, and Wei Gao are co-first authors.

\section{Disclosure}

The authors report no conflicts of interest in this work.

\section{References}

1. Pan Y, Liu X, Huang Y. Small interfering RNA-mediated knockdown of twist attenuates the aggressive phenotypes of human endometrial carcinoma Ishikawa cells. Exp Ther Med. 2017;14(6):5647-5651.

2. Tekade RK, Tekade M, Kesharwani P, D'Emanuele A. RNAi-combined nano-chemotherapeutics to tackle resistant tumors. Drug Discov Today. 2016;21(11):1761-1774.

3. Mitragotri S, Burke PA, Langer R. Overcoming the challenges in administering biopharmaceuticals: formulation and delivery strategies. Nat Rev Drug Discov. 2014;13(9):655-672.

4. Whitehead KA, Langer R, Anderson DG. Knocking down barriers: advances in siRNA delivery. Nat Rev Drug Discov. 2009;8(2):129-138.

5. Theodossiou TA, Goncalves AR, Yannakopoulou K, Skarpen E, Berg K. Photochemical internalization of tamoxifens transported by a "Trojanhorse" nanoconjugate into breast-cancer cell lines. Angew Chem Int Ed Engl. 2015;54(16):4885-4889.

6. Boussif O, Lezoualc'h F, Zanta MA, et al. A versatile vector for gene and oligonucleotide transfer into cells in culture and in vivo: polyethylenimine. Proc Natl Acad Sci U S A. 1995;92(16):7297-7301.

7. Lu Y, Lin Y, Chen Z, et al. Enhanced endosomal escape by light-fueled liquid-metal transformer. Nano Lett. 2017;17(4):2138-2145. 
8. Li S, Yuan H, Chen H, et al. Cationic poly(p-phenylene vinylene) materials as a multifunctional platform for light-enhanced siRNA delivery. Chem Asian J. 2016;11(19):2686-2689.

9. Kim H, Lee D, Kim J, Kim TI, Kim WJ. Photothermally triggered cytosolic drug delivery via endosome disruption using a functionalized reduced graphene oxide. ACS Nano. 2013;7(8):6735-6746.

10. Li L, Zhang R, Lu C, et al. In situ synthesis of NIR-light emitting carbon dots derived from spinach for bio-imaging applications. J Mater Chem B. 2017;5(35):7328-7334.

11. Weissleder R. A clearer vision for in vivo imaging. Nat Biotechnol. 2001; 19(4):316-317.

12. Wang X, Zhang J, Wang Y, et al. Multi-responsive photothermalchemotherapy with drug-loaded melanin-like nanoparticles for synergetic tumor ablation. Biomaterials. 2016;81:114-124.

13. Yang Y, Yang Y, Xie X, et al. Dual-modified liposomes with a twophoton-sensitive cell penetrating peptide and NGR ligand for siRNA targeting delivery. Biomaterials. 2015;48:84-96.

14. Lu W, Zhang G, Zhang R, et al. Tumor site-specific silencing of NFkappaB p 65 by targeted hollow gold nanosphere-mediated photothermal transfection. Cancer Res. 2010;70(8):3177-3188.

15. Chen G, Ma B, Xie R, Wang Y, Dou K, Gong S. NIR-induced spatiotemporally controlled gene silencing by upconversion nanoparticle-based siRNA nanocarrier. J Control Release. Epub 2017 Dec 27.

16. Kim H, Kim WJ. Photothermally controlled gene delivery by reduced graphene oxide-polyethylenimine nanocomposite. Small. 2014;10(1): $117-126$.

17. Kim J, Kim H, Kim WJ. Single-layered MoS2-PEI-PEG nanocompositemediated gene delivery controlled by photo and redox stimuli. Small. 2016;12(9):1184-1192.

18. Repenko T, Rix A, Nedilko A, et al. Strong photoacoustic signal enhancement by coating gold nanoparticles with melanin for biomedical imaging. Adv Funct Mater. 2018;28(7):1705607.

19. Zhang R, Cheng K, Antaris AL, et al. Hybrid anisotropic nanostructures for dual-modal cancer imaging and image-guided chemo-thermo therapies. Biomaterials. 2016;103:265-277.

20. Xu W, Sun J, Li L, Peng X, Zhang R, Wang B. Melanin-manganese nanoparticles with ultrahigh efficient clearance in vivo for tumortargeting T1 magnetic resonance imaging contrast agent. Biomater Sci. 2018;6(1):207-215.
21. Kim MA, Yoon SD, Lee CM. A drug release system induced by near infrared laser using alginate microparticles containing melanin. Int $J$ Biol Macromol. 2017;103:839-844.

22. Liu Y, Ai K, Liu J, Deng M, He Y, Lu L. Dopamine-melanin colloidal nanospheres: an efficient near-infrared photothermal therapeutic agent for in vivo cancer therapy. Adv Mater. 2013;25(9):1353-1359.

23. Fan Q, Cheng $\mathrm{K}, \mathrm{Hu} \mathrm{X}$, et al. Transferring biomarker into molecular probe: melanin nanoparticle as a naturally active platform for multimodality imaging. J Am Chem Soc. 2014;136(43):15185-15194.

24. Sharker SM, Lee JE, Kim SH, et al. $\mathrm{pH}$ triggered in vivo photothermal therapy and fluorescence nanoplatform of cancer based on responsive polymer-indocyanine green integrated reduced graphene oxide. Biomaterials. 2015;61:229-238.

25. Zhang R, Fan Q, Yang M, et al. Engineering melanin nanoparticles as an efficient drug-delivery system for imaging-guided chemotherapy. Adv Mater. 2015;27(34):5063-5069.

26. Varkouhi AK, Scholte M, Storm G, Haisma HJ. Endosomal escape pathways for delivery of biologicals. J Control Release. 2011;151(3):220-228.

27. Bieber T, Meissner W, Kostin S, Niemann A, Elsasser HP. Intracellular route and transcriptional competence of polyethylenimine-DNA complexes. J Control Release. 2002;82(2-3):441-454.

28. Salzano G, Riehle R, Navarro G, Perche F, De Rosa G, Torchilin VP. Polymeric micelles containing reversibly phospholipid-modified antisurvivin siRNA: a promising strategy to overcome drug resistance in cancer. Cancer Lett. 2014;343(2):224-231.

29. Fan B, Kang L, Chen L, et al. Systemic siRNA delivery with a dual $\mathrm{pH}$-responsive and tumor-targeted nanovector for inhibiting tumor growth and spontaneous metastasis in orthotopic murine model of breast carcinoma. Theranostics. 2017;7(2):357-376.

30. Guo C, Chen Y, Gao W, et al. Liposomal nanoparticles carrying antiIL6R antibody to the tumour microenvironment inhibit metastasis in two molecular subtypes of breast cancer mouse models. Theranostics. 2017;7(3):775-788.

31. National Research Council (US) Committee for the Update of the Guide for the Care and Use of Laboratory Animals. Guide for the Care and Use of Laboratory Animals. Vol. 327. 8th ed. Washington, DC: National Academies Press; 2011:963-965.
International Journal of Nanomedicine

\section{Publish your work in this journal}

The International Journal of Nanomedicine is an international, peerreviewed journal focusing on the application of nanotechnology in diagnostics, therapeutics, and drug delivery systems throughout the biomedical field. This journal is indexed on PubMed Central, MedLine, CAS, SciSearch $®$, Current Contents ${ }^{\circledR} /$ Clinical Medicine,

\section{Dovepress}

Journal Citation Reports/Science Edition, EMBase, Scopus and the Elsevier Bibliographic databases. The manuscript management system is completely online and includes a very quick and fair peer-review system, which is all easy to use. Visit http://www.dovepress.com/ testimonials.php to read real quotes from published authors. 